\title{
Femtosecond Electron Diffraction: Direct Probe of Ultrafast Structural Dynamics in Metal Films
}

\author{
SHOUHUA NIE, XUAN WANG, JUNJIE LI, RICHARD CLINITE, AND JIANMING CAO* \\ Physics Department and National High Magnetic Field Laboratory, Florida State University, Tallahassee, Florida 32310
}

\author{
KEY WORDS FED; coherent phonon; electronic thermal expansion; electronic Grüneisen \\ parameter
}

\begin{abstract}
Femtosecond electron diffraction is a rapidly advancing technique that holds a great promise for studying ultrafast structural dynamics in phase transitions, chemical reactions, and function of biological molecules at the atomic time and length scales. In this paper, we summarize our development of a tabletop femtosecond electron diffractometer. Using a delicate instrument design and careful experimental configurations, we demonstrate the unprecedented capability of detecting submilli-ångström lattice spacing change on a subpicosecond timescale with this new technique. We have conducted an in-depth investigation of ultrafast coherent phonon dynamics induced by an impulsive optical excitation in thin-film metals. By probing both coherent acoustic and random thermal lattice motions simultaneously in real time, we have provided the first and unambiguous experimental evidence that the pressure of hot electrons contributes significantly to the generation of coherent acoustic phonons under nonequilibrium conditions when electrons and phonons are not thermalized. Based on these observations, we also propose an innovative approach to measure the electronic Grüneisen parameter in magnetic materials at and above room temperature, which provides a way to gain new insights into electronic thermal expansion in ferromagnetic transition metals. Microsc. Res. Tech. 72:131-143, $2009 . \quad$ @ 2009 Wiley-Liss, Inc.
\end{abstract}

\section{INTRODUCTION}

Direct and real-time monitoring and understanding of atomic-level structural dynamics are important frontiers of scientific research and application. The importance of these research activities is underlined by the fact that structural changes in materials govern many fundamental processes in nature, such as phase transitions, surface dynamics, and chemical and biological reactions. Although these processes span a broad temporal range of milliseconds to hundreds of femtoseconds, ultimately all of them are driven by the motions of atoms on the time scale of a single vibrational period of 1 picosecond or less (Schoenlein et al., 2001; Sundström, 1996; Zewail, 2000). A real-time and atomiclevel view of these dynamics holds the potential to fully reveal the physical mechanisms and the structurefunction correlations that would otherwise be impossible to extract.

Both ultrafast X-ray and electron diffraction carry the capacity and potential for capturing the atomiclevel details of structural dynamics, and each of these two techniques has unique strengths and challenges that make them complementary. We use ultrafast electron pulses to probe structural dynamics for several reasons: First, at the commonly used $30-100 \mathrm{keV}$ beam energy, the atomic elastic scattering cross-section of electrons is close to five orders of magnitude greater than that of X-rays (Bonham and Fink, 1974; Hubbell and Overbo, 1979), leading to a much stronger diffraction signal and less damage to the sample for a given dosage. Second, the scattering length of electrons closely matches the optical penetration depth of the pump laser for most samples, which ensures a more uniform excitation of the probed region. In addition, the experimental setup is very compact and tabletop, and requires neither state-of-the-art high-intensity lasers nor large-scale accelerators. The latter feature is perhaps most appealing to research groups at universities and research institutes because the setup can be readily integrated into an ordinary lab setting.

Since the pioneering work in early 1980s (Mourou and Williamson, 1982; Williamson et al., 1984), these superior capabilities and potential had not been fully appreciated until recent progress in the field of timeresolved electron diffraction (Baum and Zewail, 2007; Cao et al., 2003; Dudek and Weber, 2001; Hebeisen et al., 2006; Siwick et al., 2003; Srinivasan et al., 2003; Williamson et al., 1993; Zewail, 2006). These breakthroughs make it possible to use ultrafast electron diffraction to study a myriad array of structural processes that can be photoexcited in real time. In this paper, we provide an overview of our development of a tabletop femtosecond electron diffractometer and its application to study the ultrafast structure dynamics in thin metal films induced by femtosecond optical excitations, with special emphasis on the direct probe of coherent and

*Correspondence to: . E-mail: jcao@magnet..fsu.edu

Received 29 February 2008; accepted in revised form 7 August 2008

Contract grant sponsors: Florida State University, National High Magnetic Field Laboratory, and National Science Foundation; Contract grant numbers: DMR-0305519 and DMR-0606431.

DOI 10.1002/jemt.20666

Published online 7 January 2009 in Wiley InterScience (www.interscience. wiley.com). 


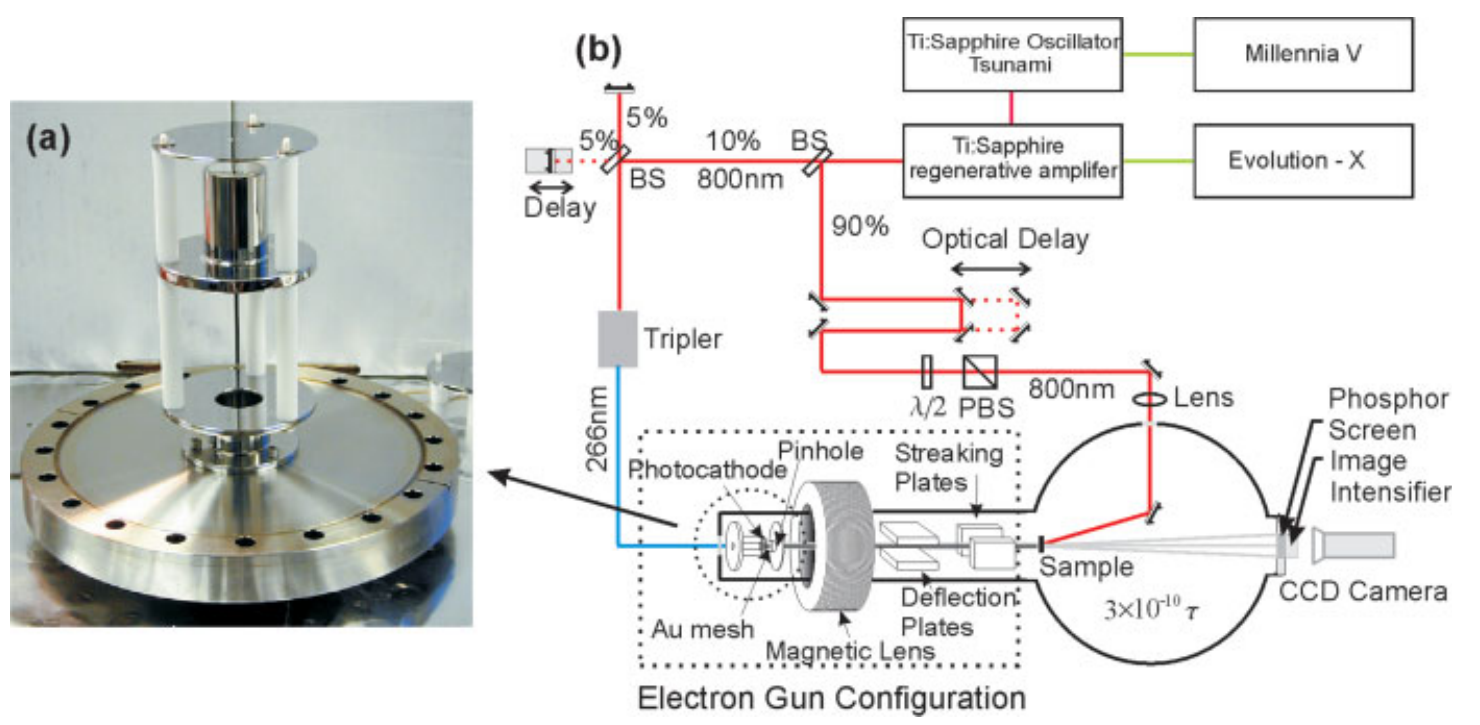

Fig. 1. (a) The second-generation electron gun. (b) Schematic of femtosecond electron diffraction setup. [Color figure can be viewed in the online issue, which is available at www.interscience.wiley.com.]

thermal lattice motions, the mechanism of coherent phonon generation, and a new method of measuring the electronic Grüneisen parameter.

\section{Experimental Setup and Considerations}

Femtosecond electron diffraction (FED), similar to time-resolved optical measurements, uses femtosecond optical pulses and the pump-probe technique. In a typical FED measurement, a femtosecond optical pulse is first split into two through optical means. One pulse is directed to a sample to initiate the structural dynamics, while the other is converted to an ultrashort electron pulse via photoemission. The electron pulse is then used to record the transient structures by taking snapshots of diffraction patterns at various delay times. Because the pump and the electron generating laser pulses are originally ripped from the same optical pulse, they can be precisely synchronized and delayed to within a few femtoseconds' accuracy by varying the difference of their optical path lengths. Our FED apparatus consists of four major components: an amplified femtosecond laser system, a femtosecond pulsed electron gun, a diffraction imaging system, and a streak camera, as shown in Figure 1(b).

To capture atomic-level structural dynamics in real time, the probe pulse duration must be shorter than the fundamental timescale of a single vibrational pe$\operatorname{riod}(100 \mathrm{fs}-1 \mathrm{ps})$ so that the atomic motions can be frozen and render clean snapshots of diffraction patterns. Additionally, the diffraction data recorded at each time point must retain a sufficient signal to noise ratio (SNR) to reveal atomic-level details of transient structures at a spatial resolution better than a fraction of bond length or Bravais lattice spacing. Although each of these requirements can be routinely fulfilled individually, meeting both requirements simultaneously presents an enormous challenge that can be largely attributed to the extremely low duty cycle inherited in time-resolved measurements using ultrashort electron pulses. For example, a measurement using 1-ps elec- tron pulses running at a $1-\mathrm{kHz}$ repetition rate has only $1 \mathrm{~ns}$ of actual data integration time every second, with a duty cycle of $10^{-9}$. This huge deficit in signal integration time can be compensated for in part by boosting the electron beam intensity. However, higher electron density leads to longer electron pulse width owing to the space charge effect. Consequently, the increase of diffraction signal comes at the cost of reduced temporal resolution and vice versa. For most of ultrafast electron diffractometers currently used, the maximum number of electrons per pulse is usually limited to a few thousand or less for a subpicosecond pulse duration at a beam energy in the range of $30-100 \mathrm{keV}$. Therefore, delicate instrument design and careful experimental configurations are required to accomplish simultaneous optimal spatial and temporal resolution.

In general, the performance of a FED instrument depends jointly on the characteristics of the electron gun (its monochromaticity, beam size, and collimation angle), the quality of imaging system (its detective quantum efficiency, dark noise, point spread function, gain uniformity and dynamic range), and experimental SNR. Its overall temporal resolution is set by several factors and can be estimated as follows:

$$
\tau_{\text {total }}=\left(\tau_{\text {pump }}^{2}+\tau_{\text {probe }}^{2}+\tau_{\text {mismatch }}^{2}\right)^{1 / 2}
$$

where $\tau_{\text {pump }}$ and $\tau_{\text {probe }}$ are pump laser and probe electron pulse durations, and $\tau_{\text {mismatch }}$ is the temporal smearing introduced by their different velocities and geometry arrangements. In the following sections, we discuss in more detail how to construct, control, and configure each component to achieve the required spatiotemporal resolution.

\section{Femtosecond Laser System and Optical Components}

A commercial diode-pumped regenerative amplified Ti:sapphire laser system is used in the FED setup. It outputs sub-50-fs laser pulses with a wavelength tun- 
ing range of $780-820 \mathrm{~nm}$ and at an adjustable repetition rate of a few $\mathrm{Hz}$ to up to $10 \mathrm{kHz}$. The output pulse energy is about $900 \mu \mathrm{J}$ at a $1-\mathrm{kHz}$ repetition rate. In a typical FED experiment, the output laser pulses at 800-nm wavelength are first divided into pump and probe pulses by a beam splitter. The pump pulses, containing $\sim 90 \%$ of original beam energy, are directed through a precision linear translation stage and used to initiate the structural dynamics. The remaining $10 \%$ of the optical pulses are sent through a frequency tripler. The tripled femtosecond pulses, with photon energy of $4.65 \mathrm{eV}$, are converted to femtosecond electron pulses via photoemission and used to record the temporal evolutions of structural changes by taking snapshots of transmission diffraction patterns. The delay time between the excitation optical and the probe electron pulses is controlled by varying the relative optical path difference between the two beams.

Several optical elements are also added in the setup to perform specific functions for FED measurements. A half wave-plate and a polarized beam splitter are inserted in the pump beam path for the fine tuning of pump beam energy. In addition, a Michelson interferometer was set up to generate two excitation laser pulses separated by a well-defined time delay. In the interferometer, each incoming optical pulse was divided into two equal parts by a beam splitter and their time delay after exiting the interferometer was adjusted by changing the length difference between the two arms. This interferometer can be inserted either in the pump optical path for coherent control of structural dynamics or in the probe optical path before the tripler for the streaking measurements of electron pulse duration [Fig. 1(b)].

\section{Femtosecond Electron Gun: Femtosecond Electron Pulse Generation and Propagation}

The femtosecond electron gun is the most critical component of an FED apparatus. Its performance (temporal duration, beam size and divergence, energy spread, and their correlations with the number of electrons per pulse) determines the overall temporal and spatial resolution of the diffractometer. The configuration of our DC electron gun is shown in Figure 1. It is composed of a negatively biased photocathode (PC), an extraction assembly with a grounded gold mesh with a $150-\mu \mathrm{m}$ pinhole behind it, a magnetic lens, a set of deflection plates, and a pair of streaking plates. Femtosecond electron pulses, generated via photoemission by back-illuminating a PC, are accelerated to $60 \mathrm{keV}$ in a high electric field of $12 \mathrm{MV} / \mathrm{m}$ between the $\mathrm{PC}$ and the gold mesh. After passing through the pinhole, the electron beam is collimated by a magnetic lens and positioned to the sample to perform diffraction measurements.

The electron pulse broadening during its transit from PC to sample is the most critical factor affecting the temporal resolution. It can usually be considered in two separate regions: the initial acceleration (high electric field region) from $\mathrm{PC}$ to extraction mesh and the subsequent drifting to a sample target. Owing to the very short electron transit time from the PC to extraction mesh (about $75 \mathrm{ps}$ for $5 \mathrm{~mm}$ ), the pulse broadening results mainly from the transit time difference of electrons with different kinetic energies. Accordingly, the pulse duration is determined by photoelectron energy spread $\Delta \varepsilon$ and the strength of the extraction electric field $E$ (Schelev et al., 1971):

$$
\Delta t_{\mathrm{PC}}=\frac{\sqrt{2 m_{e} \Delta \varepsilon}}{e E}
$$

For a DC gun without any pulse compression component, $\Delta t_{\mathrm{PC}}$ is the shortest pulse duration that can be obtained. Apparently, narrower photoelectron energy spread and higher extraction field are favored over shorter pulse generation. Using a thermally evaporated $40-\mathrm{nm} \mathrm{Ag}$ film as photocathode photoexcited at $266 \mathrm{~nm}$ in our electron gun, the typical energy spread is $\sim 0.6 \mathrm{eV}$ (Srinivasan et al., 2003). To achieve the highest possible extraction field, we have taken special care in building the electrodes exposed to the high electric field, avoiding sharp edges and polishing the surfaces to mirror-shine flat. We find this step critical for removing irregularities and asperities that can generate electric arcing. For the current electron gun design, we are able to maintain a $12-\mathrm{kV} / \mathrm{mm}$ extraction field. The corresponding $\Delta t_{\mathrm{PC}}$ is about $220 \mathrm{fs}$. Further shortening of $\Delta t_{\mathrm{PC}}$ can be achieved by minimizing $\Delta \varepsilon$, maximizing extraction field, and including a pulse compression in the gun design. An excellent example is the recent development of relativistic electron diffraction at megaelectron volt beam energy. By using a radio frequency cavity that incorporates both a pulsed highextraction electric field and a bunch compression scheme, a single electron pulse containing more than a million electrons with subps pulse duration has been generated, making it possible to conduct a real singleshot measurement of transient structure (Hastings et al., 2006; Wang et al., 2006).

In the beam-drifting region, the dominant broadening comes from the mutual repulsive interactions among electrons bunched in a short pulse-the so-called space charge effect. This broadening is unavoidable in the FED measurements demanding high electron density; therefore, minimizing and controlling the spacecharge effect is essential to maintain subpicosecond time resolution. Extensive studies have been performed, and several models have been proposed (Qian and Elsayed-Ali, 2002; Reed, 2006; Siwick et al., 2002) to analyze the space-charge broadening as a function of electron density, propagation time, and electron beam size. In general, the key to achieving a short electron pulse is reducing the electron numbers per pulse and/ or minimizing the beam propagation time to sample. For a given integration time, however, lower electron beam intensity results in a poor SNR of diffraction data and hence diminishes the spatial resolution and sensitivity in detection of transient structural changes. It should be noted that this SNR deficit, in most cases, cannot be recovered by merely increasing the data acquisition time, because a typical FED experiment has already been stretched to run continuously for about a week owing to the extremely low duty cycle.

In this regard, the preferable method is to minimize the drifting time of the electron pulse either by shortening the distance from the PC to the sample or by increasing the electron kinetic energy. In the former case, the electron pulse travel distance of $\sim 2.4 \mathrm{~cm}$ has 
been achieved (Dwyer et al., 2006). This extremely compact design can produce subpicosecond electron pulses with $\sim 10^{4}$ electrons per packet, which makes it possible to study irreversible structural transitions in a nearly single-shot measurement.

Our approach is increasing the electron beam energy while keeping only fundamental electron optics to shorten the electron pulse propagation time. The second-generation electron gun, with $60-\mathrm{keV}$ beam energy and $12-\mathrm{MV} / \mathrm{m}$ extraction field, can deliver an electron pulse shorter than 400 fs containing 1,000 electrons at the sample location (Wang et al., submitted for publication). In our more recent third-generation electron gun, we further increase the beam energy up to 100 $\mathrm{keV}$. This new electron gun is expected to deliver twice as many electrons per pulse while maintaining the same pulse duration.

\section{Temporal Degradation Owing to Diffraction Geometry}

Owing to their velocity mismatch and geometrical arrangements, the electron and laser pulses cannot be synchronized across the entire probed region. This asynchronization deteriorates the overall temporal resolution. In certain conditions-such as reflection highenergy electron diffraction geometry used for surface studies and gas phase experiments with typical molecular beam sizes of several hundred microns-this degradation can be a few picoseconds and longer, preventing the subpicosecond diffraction measurements (Williamson and Zewail, 1993).

To minimize this degradation, we use transmission electron-diffraction geometry with a near-collinear arrangement of pump laser and probe electron beams. For the ultrathin samples with thickness less than 100 $\mathrm{nm}$ used in electron-transmission diffraction, the temporal smearing from velocity mismatch is less than one femtosecond. Therefore, the degradation associated with the geometrical arrangement of optical and electron pulses is a dominant factor in our FED experiment. By reducing the beam cross-angle to less than $10^{\circ}$ (Fig. 2), this time smearing from geometry mismatch is limited to a value less than 200 fs for an electron beam diameter of $350 \mu \mathrm{m}$. In the reflection highenergy electron diffraction geometry, this temporal degradation can be reduced from $10 \mathrm{ps}$ to subpicosecond by spatially tilting the pump optical pulse such that the initiating laser pulse and probe electron pulse arrive at each point of the sample simultaneously (Baum et al., 2007; Baum and Zewail, 2006).

\section{Two-dimensional Single Electron Detector}

To offset the extremely low duty cycle and weak overall diffraction intensity, the diffraction imaging system must have the capability of single electron detection at a low noise level. The detector used in our FED consists of a linear chain of a phosphor screen, an image intensifier, and a thermoelectrically cooled charge-coupled device camera. It has a detective quantum efficiency of $\sim 75 \%$, which is calibrated with a homemade Faraday cup, and has a point spread function less than $40 \mu \mathrm{m}$ ( $25 \mathrm{lp} / \mathrm{mm})$.

Most of the experimental results shown here were obtained with this detector and the second-generation

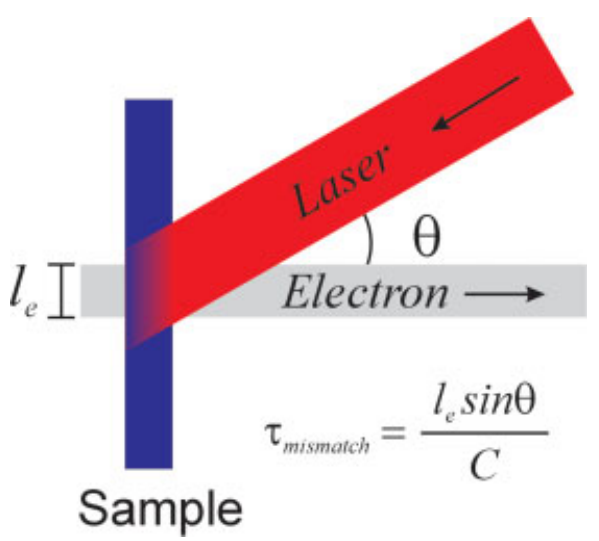

Fig. 2. The degradation of temporal resolution in femtosecond electron diffraction owing to the geometrical arrangement of laser and electron pulses. The arrows show the directions of the pulses. The temporal degradation of our setup is $\sim 200 \mathrm{fs}$. [Color figure can be viewed in the online issue, which is available at www.interscience. wiley.com.]

60-keV electron gun. For a single diffraction pattern of a 20 -nm thick Al film recorded with roughly $2 \times 10^{7}$ electrons ( $\sim 20$-s integration time), we are able to detect less than $0.5 \%$ lattice spacing change by using a standard diffraction data analysis scheme. This detection sensitivity can be further enhanced by longer data integration time $(t)$ with improved SNR $(\mathrm{SNR} \propto \sqrt{t})$. Using diffraction data averaged over twenty sets of such diffraction patterns, we are able to detect less than $0.02 \%$ lattice spacing change (submilli-ångström) (Park et al., 2005b).

\section{MATERIALS AND METHODS}

Similar to conventional transmission electron microscopy (TEM), transmission geometry for ultrafast diffraction measurement also uses a thin sample. By using a sample with a thickness comparable to the elastic mean free path of probe electrons $(50 \mathrm{~nm}$ in $\mathrm{Al}$ for a $60-\mathrm{keV}$ electron beam), we can complete the diffraction data analysis using only the kinematical theory (Reimer, 1997), because on average each probe electron suffers less than one collision through the film.

An added benefit of ultrathin metal films is the realization of an ultrafast and homogenous excitation across the entire sample thickness, which is a condition critical for accurate measurement of structural dynamics with FED. For most metals, although the penetration depth of pump optical pulses is only a few nanometers, the transport of hot electrons is expected to be quasi-ballistic at the speed of Fermi velocity of $1 \mathrm{~nm} / \mathrm{fs}$, because the mean free path of photoexcited electrons is on the same order of magnitude of film thickness (Hohlfeld et al., 2000). Thus, the initial nonuniform excitation as generated by photoabsorption can be counterbalanced by the ultrafast transport of photoexcited hot electrons. As a result, a $100 \mathrm{~nm}$ film can be considered homogenously excited in about $100 \mathrm{fs}$. 


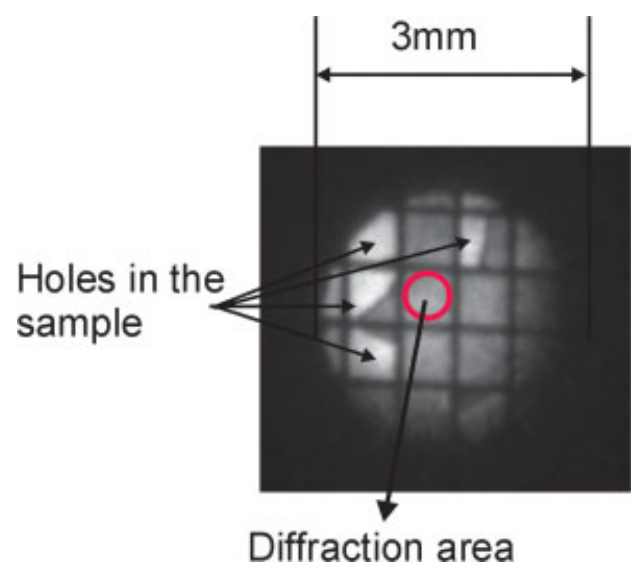

Fig. 3. Electron shadow image of a thin Al film on a 50-mesh TEM grid taken with an unfocused electron beam. The circled region was selected for the femtosecond electron diffraction measurement. [Color figure can be viewed in the online issue, which is available at www. interscience.wiley.com.]

\section{Sample Preparation}

To make polycrystalline metal films, we first cleave a 1 -in. $\times 1$-in. $\times 1$-in. $\mathrm{NaCl}$ single crystal into many $1 \mathrm{~cm}$ $\times 1 \mathrm{~cm} \times 2 \mathrm{~mm}$ slices with mirror-like surfaces as substrates. We then coat metal films (Al, for example) onto these $\mathrm{NaCl}$ substrates using a high-vacuum thermal evaporator with desired thickness measured by an in situ thickness monitor. After that, the thin-metal films are transferred to standard TEM grids by floating and picking them onto the grids in distilled water. A typical sample picture is shown in Figure 3. The FED measurements are conducted inside an ultrahigh vacuum chamber with base pressure less than $3 \times 10^{-10}$ torr by mounting the sample grids on a customized ultrahigh vacuum TEM sample holder with a temperature tuning range of $80-600 \mathrm{~K}$ at $0.1 \mathrm{~K}$ accuracy.

\section{Determination of Time Zero}

Accurate and independent determination of the time zero point, where optical and electron pulses temporally overlap, is crucial for FED experiments (Park et al., 2005a), particularly for the study of irreversible structural dynamics, where strong laser excitationinduced sample damage prevents the extensive search of time zero. In the case of low optical excitation and reversible experiments, accurate pinning of time zero helps to narrow down the scanned temporal range and consequently leads to an increased SNR in a given experimental time span. The determination of time zero is desired to have subpicosecond accuracy and can be conveniently conducted in situ without any additional or complicated setup.

To this end, we have developed a method similar to the photoionization-induced lensing effect that was used in gas-phase electron diffraction experiments (Dantus et al., 1994). Instead of gas molecules, a sharp silver needle is placed at the sample location as an intermediate. Following intense laser excitation at 800 $\mathrm{nm}$, the ejected electrons from needle surface via both instantaneous multiphoton photoemission and subsequent thermionic emission stay and accumulate at the
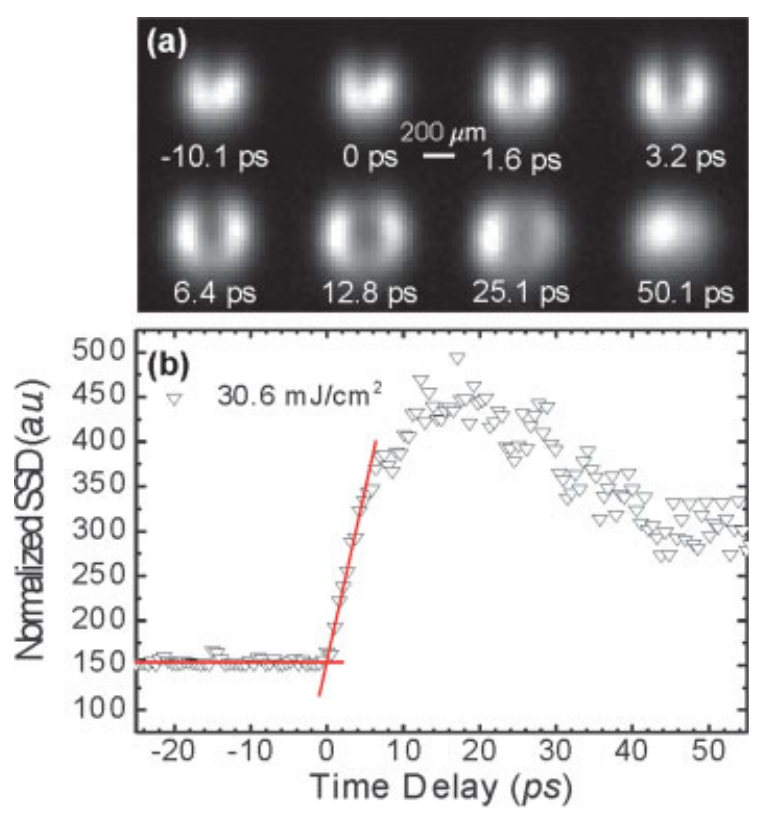

Fig. 4. (a) Temporal evolution of electron shadow images of a silver needle tip. Each image was recorded using femtosecond electron pulses containing an average of 500 electrons per pulse and with an integration time of $0.5 \mathrm{~s}$. The excitation laser fluence was $30.6 \mathrm{~mJ} /$ $\mathrm{cm}^{2}$. (b) Temporal resolution of SSD curve. Time zero is defined by the crossing point of two linear fittings, one for the background (horizontal) before laser excitation and the other for the rising edge. [Color figure can be viewed in the online issue, which is available at www. interscience.wiley.com.]

photoexcited surface area. The resulting high-density electron cloud creates a strong local electric field, which, with sufficient strength, will perturb the profile of incident electron beams, and consequently the change of beam profile should follow the dynamics of this local electric field. Hence, time zero can be accurately determined by conducting a real time and quantitative measurement of the laser-induced perturbation to the electron beam. Because the time scale for the onset of the electric field is essentially instantaneous, the onset of the beam profile change should define time zero.

Typical disturbed electron beam images as a function of pump-probe delay times are shown in Figure 4(a). These images were recorded with 500 electron pulses per image using an excitation laser fluence of $30.6 \mathrm{~mJ} /$ $\mathrm{cm}^{2}$. The average number of electrons per pulse was $\sim 500$, with the corresponding pulse length of $\sim 350 \mathrm{fs}$. As shown in these images, the original needle shadow started to expand in two dimensions following the femtosecond laser excitation. At shorter delay times, this expansion became progressively stronger with time. Then the shadow started to fade at about $25 \mathrm{ps}$ after excitation, with a simultaneous contraction of the electron beam size to its original shape. The whole process persisted more than a few hundred picoseconds.

Time zero was determined through a quantitative evaluation of the temporal evolution of these images, as displayed in Figure 4(b). The change of beam profile at each time point was calculated by summing the square of the intensity difference (SSD) in each corre- 


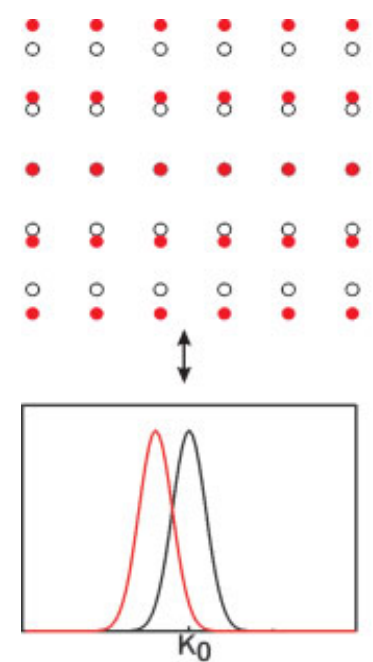

(a)

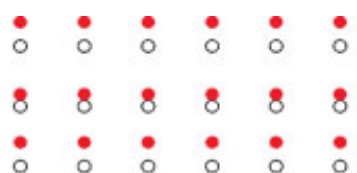

88888888

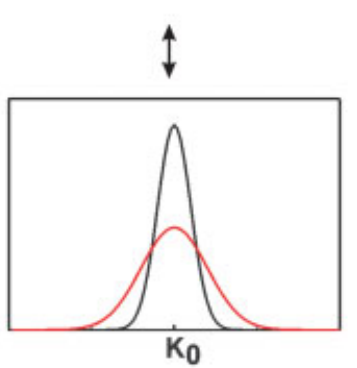

(b)

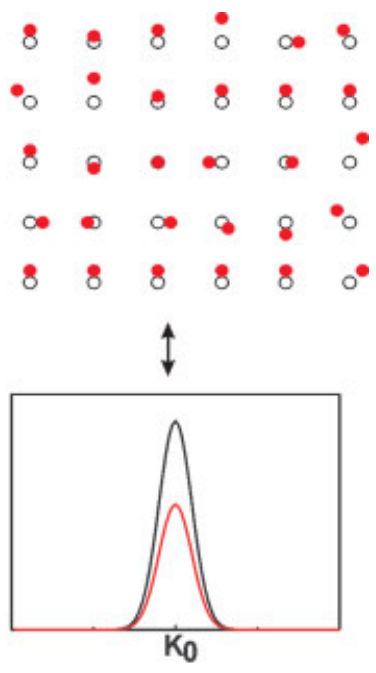

(c)

Fig. 5. Laser-induced lattice structural changes in real space and the corresponding Bragg peak alterations displayed in diffraction patterns. (a) Linear lattice expansion, (b) inhomogenous lattice spacing, and (c) lattice disorder due to heating. [Color figure can be viewed in the online issue, which is available at www.interscience.wiley.com.]

sponding pixel between two images recorded at the same time point, one with pump laser and one with pump blocked. The turning point of this SSD curve, defined here as the time zero point, is then determined as the intersection point of two linear fittings, one for the background before photoexcitation and the other for the rising edge. The typical uncertainty of the time zero point is less than $600 \mathrm{fs}$, which is calculated by convoluting the errors in two linear fittings according to standard error propagation rules.

\section{Diffraction Data Analysis}

The electron diffraction pattern of a single crystal sample contains rich information regarding how constituent atoms arrange themselves microscopically to build a crystal. The pattern indicates the type of Bravais lattice, the size and symmetry of its unit cell, and how many and what types of atoms are present and their positions in the unit cell of the crystal. According to kinematical diffraction theory, the constructive interference of diffracted electron waves (diffraction peaks) occurs according to the Bragg law:

$$
2 d_{\mathrm{hkl}} \operatorname{Sin}(\theta)=\lambda,
$$

where $d_{\mathrm{hkl}}$ is spacing between parallel lattice planes with Miller indices ( $h, k, l), \theta$ is the angle of the incident beam with respect to the diffraction plane, and $\lambda$ is incident electron De Broglie wavelength. For monoenergetic electron diffraction with fixed $\lambda$, the Bragg diffraction angle, $\theta$, gives a direct measurement of the corresponding lattice spacing.

The Bragg peak intensity provides a direct measurement of thermal lattice motions, as incoherent thermal lattice motions tend to destroy the phase coherence of the diffraction beam, resulting in a reduced Bragg peak intensity. The Bragg peak intensity attenuation as a function of lattice temperature is described by the Debye-Waller effect:

$$
I_{\mathrm{hkl}}(T)=I_{\mathrm{hkl}}(0) \exp \left[-\frac{1}{2} S_{\mathrm{hkl}}^{2} B(T)\right],
$$

where the momentum transfer $S$ is $2 \operatorname{Sin}(\theta) / \lambda$, and $B(T)$ is the Debye-Waller parameter. In Debye theory, $B(T)$ is determined as follows:

$$
B(T)=\frac{6 h^{2} T}{m k_{\mathrm{B}} \theta_{\mathrm{D}}^{2}}\left[\frac{T}{\theta_{\mathrm{D}}} \int_{0}^{\theta_{\mathrm{D}} / T} \frac{x d x}{e^{x}-1}+\frac{1}{4} \frac{\theta_{\mathrm{D}}}{T}\right],
$$

where $h$ is Planck's constant, $m$ is the mass of the atom, $k_{\mathrm{B}}$ is Boltzmann's constant, and $\theta_{\mathrm{D}}$ is the Debye temperature of the material. For a sample with $T>\theta_{\mathrm{D}}$, $B(T)$ can be well approximated to be linearly dependent on $T$-i.e., $B(T)=a_{0}+a_{1} T$. Combining the above expressions, we obtain the Bragg peak intensity attenuation induced by lattice heating as the following:

$$
I_{\mathrm{hkl}}(T+\Delta T) / I_{\mathrm{hkl}}(T)=\exp \left[-\frac{1}{2} S_{\mathrm{hkl}}^{2} a_{1} \Delta T\right] .
$$

Clearly, given the diffracted Bragg peak intensity and the Debye-Waller parameter, the lattice temperature jump $\Delta T$ can be deduced explicitly.

A snapshot of the diffraction pattern records all of this structural information at a given time point. Figure 5 summarizes the possible Bragg peak changes associated with laser-induced lattice dynamics. The laser-induced lattice spacing $(d)$ change, either contraction or expansion, can be traced by following the associated changes in Bragg peak position (diffraction angle). Inhomogeneity in the lattice spacing tends to 

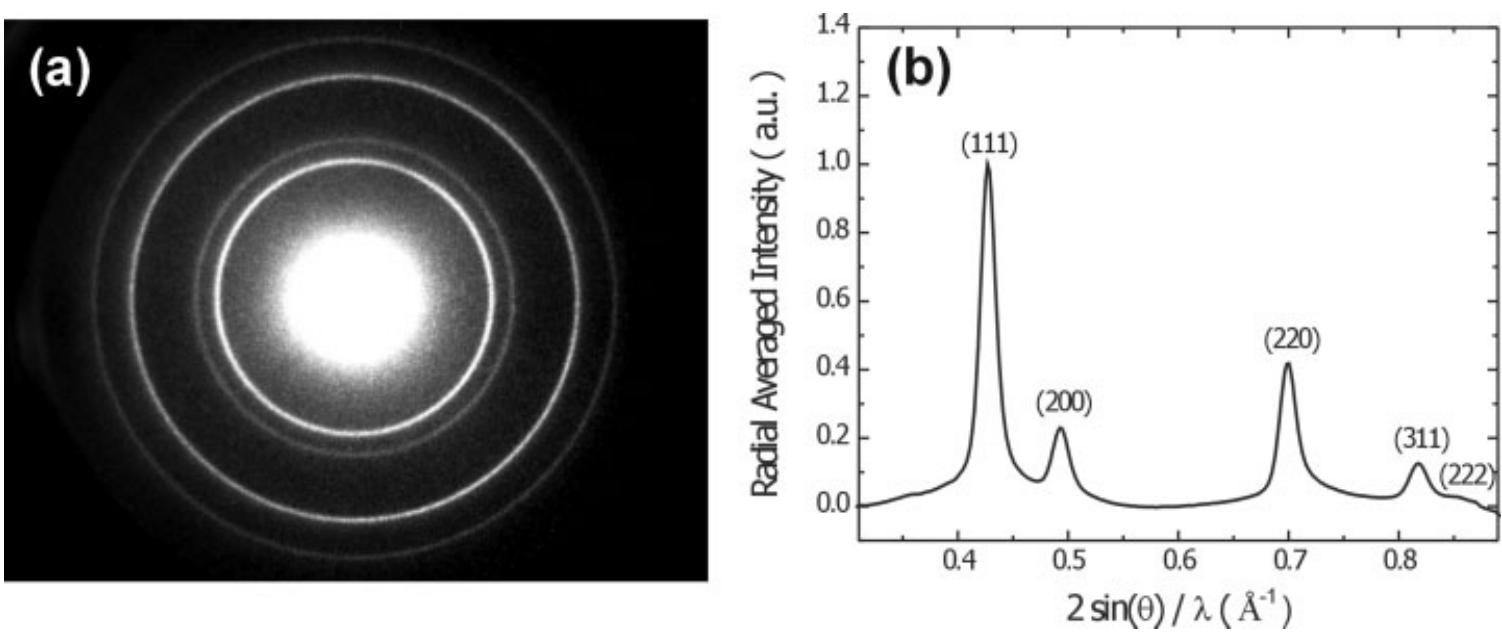

Fig. 6. (a) Typical diffraction pattern of a 20 -nm thick polycrystalline Al film recorded with $\sim 2 \times 10^{7}$ electrons. (b) The corresponding radial-averaged intensity curve after removing the background.

broaden the width of Bragg peak. Lattice disorder owing to temperature rise or loss of long-range order owing to structural phase transition result in decreased Bragg peak intensity or a complete loss of coherent Bragg peak. Therefore by measuring the position, width, and intensity of Bragg peaks in the diffraction patterns as a function of delay time, we can trace how a structure evolves with time.

A typical diffraction pattern snapsnot is shown in Figure 6(a). To extract the diffraction angle, width, and intensity of Bragg peaks from a diffraction pattern accurately, we follow a standard data analysis procedure that we developed. We first convert a two-dimensional (2-D) diffraction pattern to a diffraction intensity curve as a function of diffraction angle (momentum transfer $S=2 \sin (\theta) / \lambda)$. This conversion is performed by first finding the pixel coordinates of diffraction center (center of unscattered primary electron beam) and then radially averaging the diffraction intensity as a function of pixel distance $(r)$ from the center. This pixel distance is then converted to a momentum transfer $\left(S=\frac{2 \operatorname{Sin}(\theta)}{\lambda} \cong \frac{r}{\lambda L}\right.$ ) using the diffraction camera length $(L)$, which is calibrated with the diffraction pattern taken at room temperature from a standard commercial $\mathrm{Al}$ film. We then remove any background originating from incoherent scattering, imperfections of crystals, detector noise, and cosmic radiation. Figure $6(\mathrm{~b})$ shows the corresponding background-corrected diffraction intensity curve obtained from a 2-D diffraction pattern [Fig. $6(\mathrm{a})]$.

We then fit the intensity profile of a Bragg peak with a given function form to obtain the peak position, width, and intensity. The recorded Bragg intensity profile is a convolution of the Gaussian profile of the probe electron beam with many line-broadening effects such as imperfect focusing, wavelength dispersion, and sample strains. As a result, the analytical function forms for a best fit may vary according to these conditions. We have compared the peak position and intensity obtained by fitting a Bragg peak using different function forms (Gaussian, Lorentzian, or pseudo-Voigt) and

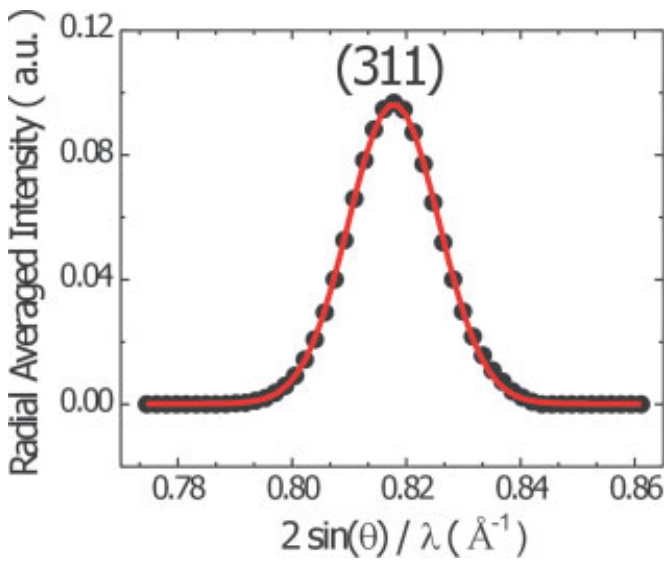

Fig. 7. A typical fit of (311) Bragg peak to a Gaussian profile. The peak center position was determined to be $0.81780 \pm 0.00003 \AA^{-1}$ (474.99 \pm 0.02 pixels). [Color figure can be viewed in the online issue, which is available at www.interscience.wiley.com.]

concluded that the difference among fitting results is insignificant because all of them cluster within one standard deviation of fitting, even though one function form gives seemingly smaller fitting uncertainty than others. A typical fitting of background-corrected (311) Bragg peak with a Gaussian function is shown in Figure 7. The uncertainty in the fitting of the peak position is usually less than $0.02 \%$.

To confirm the integrity of this diffraction data analysis, we conducted a static (nontime-resolved) measurement of the Debye-Waller parameter $a_{1}$ and linear thermal expansion coefficient $\alpha$ of $\mathrm{Al}$ by varying sample temperature. The results are shown in Figure 8. By measuring the Bragg peak intensity and position as a function of temperature and performing a linear fit, we obtained $a_{1}=(6.2 \pm 0.8) \times 10^{-5} \mathrm{~nm}^{2} \mathrm{~K}^{-1}$ and $\alpha$ $=(23.0 \pm 2.5) \times 10^{-6} \mathrm{~K}^{-1}$, respectively. Both values are in excellent agreement with those reported in the liter- 


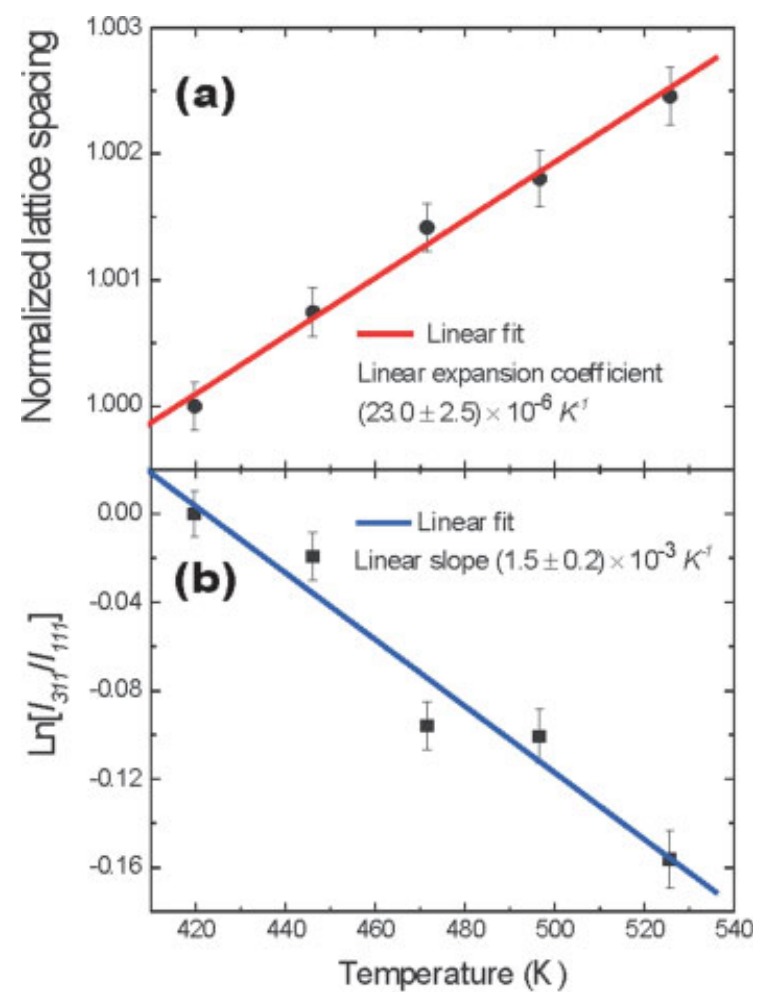

Fig. 8. Diffraction measurement of statically heated 20-nm Al film. (a) Relative lattice spacing change as a function of sample temperature. The solid line is a linear fit with linear thermal expansion coefficient $\alpha=(23.0 \pm 2.5) \times 10^{-6} / \mathrm{K}$. (b) Bragg peak intensity as a function of sample temperature. The linear temperature dependence coefficient $a_{1}$ is determined to be $(6.2 \pm 0.8) \times 10^{-5} \mathrm{~nm}^{2} / \mathrm{K}$. [Color figure can be viewed in the online issue, which is available at www. interscience.wiley.com.]

atures (Gao and Peng, 1999; Langelaan and Saimoto, 1999).

\section{RESULTS AND DISCUSSION Direct and Real-time Probe of Thermal and Coherent Lattice Motions}

The dynamics of laser-induced ultrafast heating of metals are among the first ultrafast phenomena extensively studied using a variety of time-resolved optical spectroscopic techniques (Eesley, 1986; Elsayedali et al., 1991; Sun et al., 1993; Tas and Maris, 1994). These studies have revealed that the dynamics occur over multiple steps. It starts by the ultrafast thermalization of photoexcited electrons with the cold bath of electrons below the Fermi level on a 100-fs timescale via strong electron-electron (e-e) scattering with a concurrent ballistic and diffusive transport of hot electrons from the surface into the bulk region. Electrons evolve from an initial nonequilibrium and nonuniform electron distribution as generated by photoexcitation to a Fermi-Dirac distribution with electronic temperature $\left(T_{\mathrm{e}}\right)$ that is homogenous across the entire sample thickness. At the same time, the electrons lose their energy to the lattice with temperature $T_{1}$ through electronphonon (e-ph) scattering, with a typical timescale of eph thermalization from a few picoseconds to up to 10

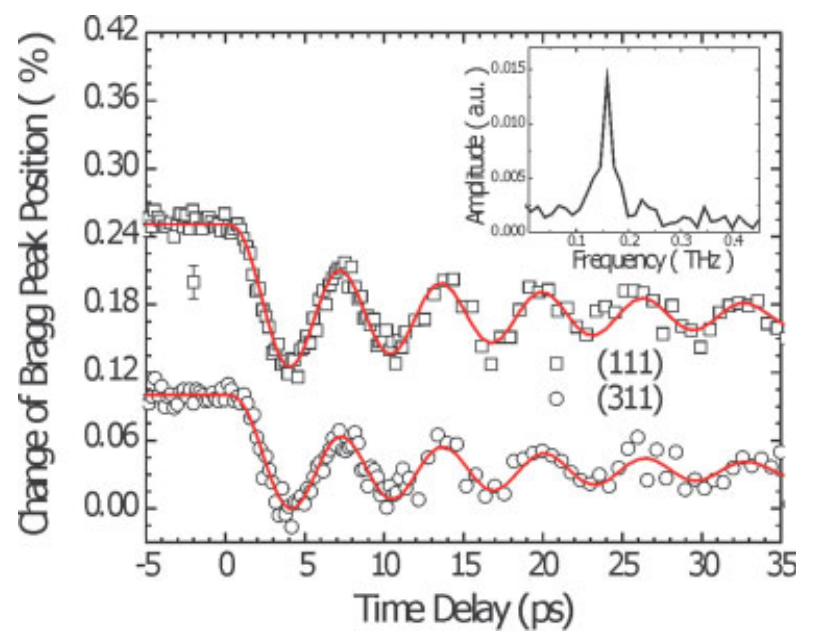

Fig. 9. Temporal evolution of (111) and (311) Bragg peak positions. The error bars represent one standard deviation from the Gaussian profile fitting. Solid lines are fits using a damped oscillator model. Inset: Fourier transform of the oscillation data of (111) peak with a single peak centered at $0.16 \mathrm{THz}$. [Color figure can be viewed in the online issue, which is available at www.interscience.wiley.com.]

ps, depending on the strength of e-ph coupling. In certain metals and under favorable excitation conditions, such as high pump laser fluence, these multi-step dynamics can be reasonably well described by a phenomenological two temperature model (TTM) (Anisimov et al., 1974). TTM treats the electrons and phonons as two separate thermalized subsystems, each with their own temperatures, and describes their interactions with an e-ph coupling constant $g$.

In metals, this heating process is ultrafast and usually completed in a few picoseconds. Thermal stress is created in a shorter time than that of certain phonon periods, and subsequently coherent acoustic waves are launched and propagate from surface into bulk. Previously, these dynamic processes have been extensively investigated using ultrafast optical measurements, albeit with indirect sensitivity to structural arrangement. We show in the following sections that by using FED with atomic-level spatiotemporal resolution, we are able to gain new physical insights into these ultrafast dynamics by probing the dynamics of both coherent and thermal lattice motions directly in real time (Park et al., 2005b).

Figure 9 depicts the temporal evolutions of (111) and (311) Bragg peak positions as a function of delay times measured with FED from a photoexcited 20-nm thick Al film. These data reveal several important features of laser-induced ultrafast lattice dynamics. First, the position of a Bragg peak displays an oscillatory motion that is centered at a newly established and reduced Bragg ring radius (expanded equilibrium lattice constant). Second, this lattice vibration starts from a maximum displacement at time zero and exhibits a nearly cosine time dependence, indicating a displacive excitation mechanism (Zeiger et al., 1992). Third, all Bragg peaks oscillate perfectly in phase with one another and within the same vibrational period. By fitting the vibration data with a damped oscillation formula (Hodak et al., 1999), we obtained nearly the same 
vibrational period of $6.4 \pm 0.5 \mathrm{ps}$ and damping time constant of $20 \pm 2 \mathrm{ps}$ for all Bragg peaks. The Fourier transform of the vibration data of the (111) peak (Fig. 9 , inset) gives a single peak with a center frequency of $0.16 \mathrm{THz}$, which is in excellent agreement with the vibration period of $6.4 \mathrm{ps}$. These data indicate that only a single mode of acoustic waves was launched. We also conducted similar measurements by varying pump laser intensity in the range of $1.4-2.3 \mathrm{~mJ} / \mathrm{cm}^{2}$ and found that the vibration amplitude is proportional to the excitation laser fluence with no observable change of vibrational period.

These Bragg peak oscillations are correlated with a breathing motion of the polycrystal $\mathrm{Al}$ film along the surface normal that is generated by an impulsive femtosecond optical excitation. After the absorption of pump optical pulse, nearly uniform heating across the entire thickness of the $\mathrm{Al}$ thin film are induced instantly, owing to the ultrafast hot electron transport (Hohlfeld et al., 2000) and strong e-ph scattering (Tas and Maris, 1994). This ultrafast heating puts the lattice in a highly stressed state by establishing a new and expanded equilibrium lattice position in a timescale shorter than the lattice response time. Under this so-called impulsive and displacive excitation (Garrett et al., 1996; Zeiger et al., 1992), a coherent lattice vibration (coherent longitudinal acoustic phonons) centered at the new equilibrium position is launched along the direction of the surface normal to release the stress. In a free-standing film with open boundaries, a one-dimensional (1-D) standing wave is formed and oscillates with a vibrational period given by $T=2 L / v$, where $L$ is film thickness, and $v$ is the longitudinal sound velocity (Cavalleri et al., 2000; Thomsen et al., 1984). In the diffraction pattern of a polycrystal film, this periodic breathing motion is exhibited as an oscillation of the Bragg ring radius. This correlation has been verified by the excellent agreement between a vibrational period of $6.4 \mathrm{ps}$ recorded with FED and that calculated using the 1-D standing wave condition with sound velocity equal to $6,420 \mathrm{~m} / \mathrm{s}$ (Lide, 2001) and measured film thickness of $20 \mathrm{~nm}$.

It is worth noting that under the current relatively low level of optical excitation and transmission diffraction geometry, the amplitude of coherent lattice vibration observed in our diffraction pattern is significantly larger than that anticipated for a thin-film that undertakes only a 1-D breathing motion along the film normal direction. Such an unusually large-amplitude coherent lattice vibration has also been observed on the surface of a photoexcited single crystal of a transition metal oxide (Gedik et al., 2007), where it was attributed to a charge-transfer-induced reconfiguration of associated chemical bonds. This attribution is evidently not the case for our observation, because no such charge-transfer channel exists in metals. Instead, we believe that this seemingly unusual, large amplitude is associated with the particular polycrystal morphology of metal films. We have developed a 2-D mass spring lattice model with the presence of grains and grain boundaries (Li et al., submitted for publication). The mass spring lattice model simulation indicates that even though the coherent lattice motions still satisfy the 1-D standing wave condition and keep the same vibrational period, the existence of grains has

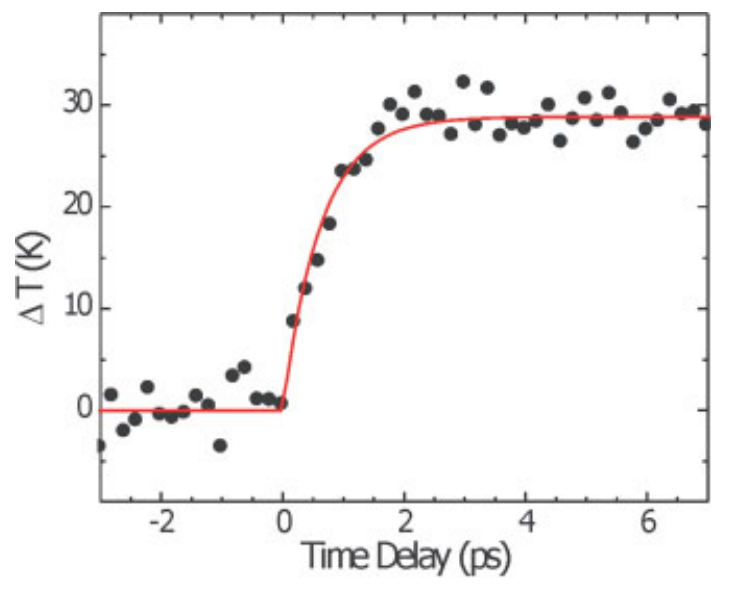

Fig. 10. Lattice temperature as a function of delay times. The solid line is a fit to the data using a single exponential function with a time constant $\tau_{\mathrm{e}-\mathrm{p}}$ of $630 \pm 90 \mathrm{fs}$. [Color figure can be viewed in the online issue, which is available at www.interscience.wiley.com.]

changed the dynamic motion mode of the metal film in response to ultrafast laser heating. Consequently, the amplitude of coherent motion in the surface parallel direction could be comparable to that of the motion along the surface normal, when the angle between grain boundaries and surface is nontrivial. Finally, it is worth pointing out that all the results presented here rely only on the temporal behavior of lattice oscillation, irrespective of the actual magnitude of its vibration.

The temporal evolution of lattice temperature measured with FED is displayed in Figure 10. These data were obtained by first calculating the (311) peak intensity and then normalizing it to that of the (111) peak for each diffraction intensity curve at a given time point. Then the normalized intensity was converted to the lattice temperature using the calibrated DebyeWaller parameter (Fig. 8). This normalization scheme using only one diffraction pattern minimizes the effect of probe electron density fluctuations, which are a major source of noise for an extended FED measurement that lasts continuously for several days. A time constant of $\tau_{\mathrm{e}-\mathrm{ph}}=630 \pm 90 \mathrm{fs}$ for the electron lattice thermalization was obtained by fitting the data with an exponential function (solid line in Fig. 10). It is important to note that the $\tau_{\mathrm{e}-\mathrm{ph}}$ value, although significantly longer than that $(\sim 200 \mathrm{fs})$ calculated through TTM simulation using widely accepted physical parameters (Tas and Maris, 1994), is in excellent agreement with the results of our and other fs optical measurements (Hostetler et al., 1999; Richardson and Spicer, 2002).

Importantly, the time it takes the lattice to reach its final equilibrium temperature $(\sim 1.9 \mathrm{ps})$ is nearly equal to one-fourth of the vibrational period $(\sim 1.6 \mathrm{ps})$. At this point the coherent lattice vibration has almost reached its new equilibrium position and gained its highest vibration energy. This observation indicates that the two modes of lattice motions-namely, coherent vibration and random thermal motions-are taking place concurrently after optical excitation. In the following 


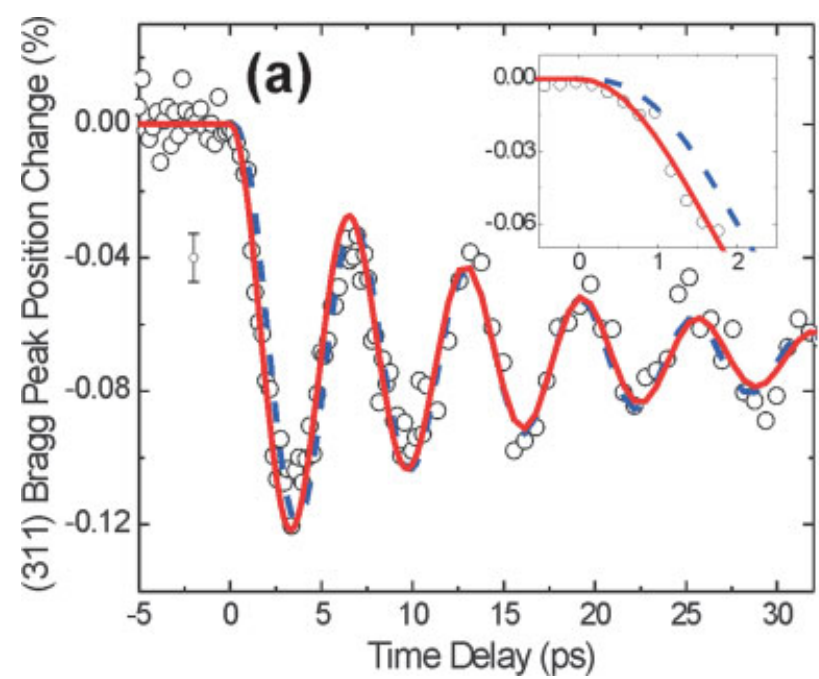

Fig. 11. (a) Temporal evolution of (311) Bragg peak positions. The solid line is a fit to the experimental data using Eqs. 7 and 9 which differentiates the electronic $\left(\sigma_{e}\right)$ and lattice $\left(\sigma_{1}\right)$ stress in time domain. The dashed line is a fit excluding electronic thermal stress, which lags behind the data with a phase shift of $\sim 18^{\circ}$. Inset: Detailed view of the

sections, we provide a detailed theoretical treatment of the correlation between these coherent and thermal lattice motions. We also discuss some new physical phenomena revealed by the FED measurements.

\section{Electronic Contribution to Coherent Phonon Generation}

The mechanism of coherent acoustic phonon generation has been under extensive study for many years (Nisoli et al., 1997; Tas and Maris, 1994). A more recent optical pump-probe measurement (Perner et al., 2000) has shown that the hot electron pressure contributes significantly to lattice expansion under ultrafast heating and nonequilibrium conditions in which electrons and phonons have not reached a thermal equilibrium. Nevertheless, a quantitative measurement of the contributions of electron and lattice heating in driving coherent acoustic phonons remains obscure. With the capacity of monitoring both coherent phonons (lattice oscillation) and their driving force (lattice temperature) simultaneously, FED has provided an unprecedented opportunity to quantitatively evaluate the significance of hot electron pressure in coherent acoustic phonon generation (Park et al., 2005a,b,c).

The roles of electron and lattice heating in driving coherent acoustic phonons were analyzed by fitting the vibration data with the differential equation of a damped harmonic oscillator. For a single frequency and low-amplitude lattice vibration, the temporal evolution of the coherent phonon field, $Q$, (displacement of coherent lattice vibration) can be well described by the following classical simple harmonic oscillator as (Kuznetsov and Stanton, 1994; Zeiger et al., 1992):

$$
\frac{d^{2} Q}{d t^{2}}+2 \beta \frac{d Q}{d t}+\omega_{0}^{2} Q=\sigma(t)
$$

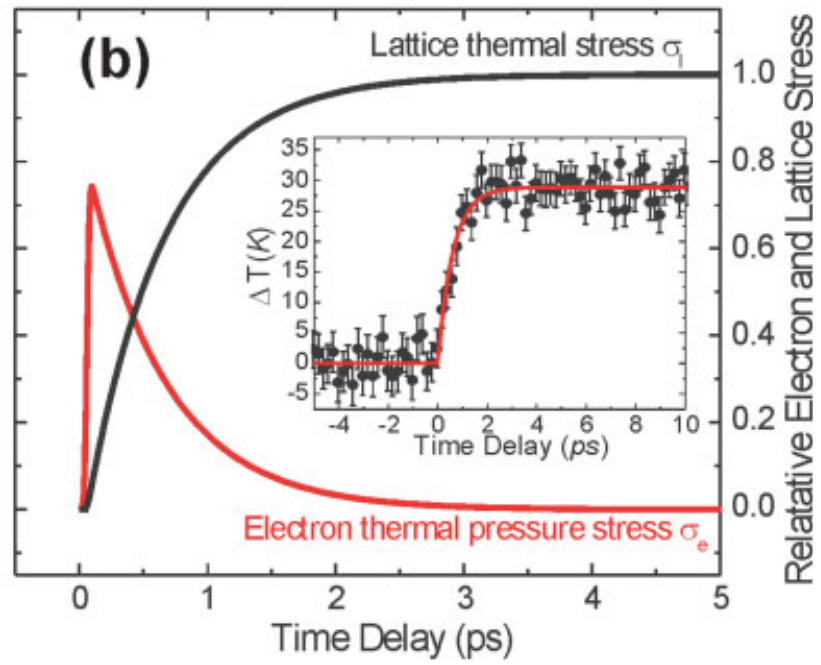

above fitting results in the temporal range of $-0.5-2.5 \mathrm{ps}$. (b) Temporal evolution of the $\sigma_{\mathrm{e}}$ and $\sigma_{1}$. Inset: The corresponding lattice temperature change as a function of delay times. [Color figure can be viewed in the online issue, which is available at www.interscience.wiley.com.]

where $\omega_{0}$ is the phonon angular frequency, $\beta$ is a phenomenological damping constant, and $\sigma(t)$ is the driving thermal stress. The thermal stress $\sigma(t)$ is induced by both electronic and lattice heating and given by (Lindenberg et al., 2000) the following:

$$
\sigma=\sigma_{\mathrm{e}}+\sigma_{1}=\gamma_{\mathrm{e}} C_{\mathrm{e}} \delta T_{\mathrm{e}}+\gamma_{1} C_{1} \delta T_{1}
$$

where $C_{\mathrm{e}}$ and $C_{1}$ are heat capacities for electrons and phonons, and $\gamma_{\mathrm{e}}$ and $\gamma_{1}$ are the corresponding Grüneisen parameters. By assuming that the total energy deposit into the probed unit volume $\left(E_{\text {total }}\right)$ by the optical pulse is conserved on the picosecond time span, we obtain $C_{\mathrm{e}} \delta T_{\mathrm{e}}+C_{1} \delta T_{1}=E_{\text {total }} g(t)$, where $g(t)$ is a Gaussian function representing the temporal profile of the 50 -fs pump optical pulse. Because $C_{\mathrm{e}}$ is much smaller than $C_{1}$, electrons transfer nearly all the absorbed photoenergy to the lattice after e-ph thermalization $\left(\delta T_{\mathrm{e}}=\right.$ $\left.\delta T_{1}\right)$. Under these conditions, the thermal energy of the lattice as a function of delay time can be expressed as $C_{1} \delta T_{1}=E_{\text {total }}\left(1-\mathrm{e}^{-t / \tau_{\mathrm{e}-\mathrm{ph}}}\right)$. Accordingly, the transient stress $\sigma$ can be uniquely determined by the e-ph coupling time constant $\left(\tau_{\mathrm{e}-\mathrm{ph}}\right)$ obtained in FED measurements as follows:

$$
\begin{aligned}
& \sigma=\sigma_{\mathrm{e}}+\sigma_{1}=-\gamma_{\mathrm{e}} E_{\text {total }}\left(g(t)-1+\mathrm{e}^{-t / \tau_{\mathrm{e}-\mathrm{ph}}}\right) \\
& -\gamma_{1} E_{\text {total }}\left(1-\mathrm{e}^{-t / \tau_{\mathrm{e}-\mathrm{ph}}}\right) .
\end{aligned}
$$

The solid curve in Fig. 12 (a) displays the least squares fit of vibration data using Eqs. (7) and (9) with values of $\gamma_{1}=2.16$, and $\gamma_{\mathrm{e}}=1.6$ (Collins et al., 1973). In the fitting, the e-ph thermalization time constant $\left(\tau_{\mathrm{e}-\mathrm{ph}}=\right.$ $600 \pm 100 \mathrm{fs})$ and time zero $\left(t_{0}=0 \pm 90 \mathrm{fs}\right)$ were fixed to the values measured using FED [Inset of Fig. 11 (b)], and the three other parameters, $\omega_{0}, \beta$, and $C$ were floated. The fitting results, with phonon angular fre- 

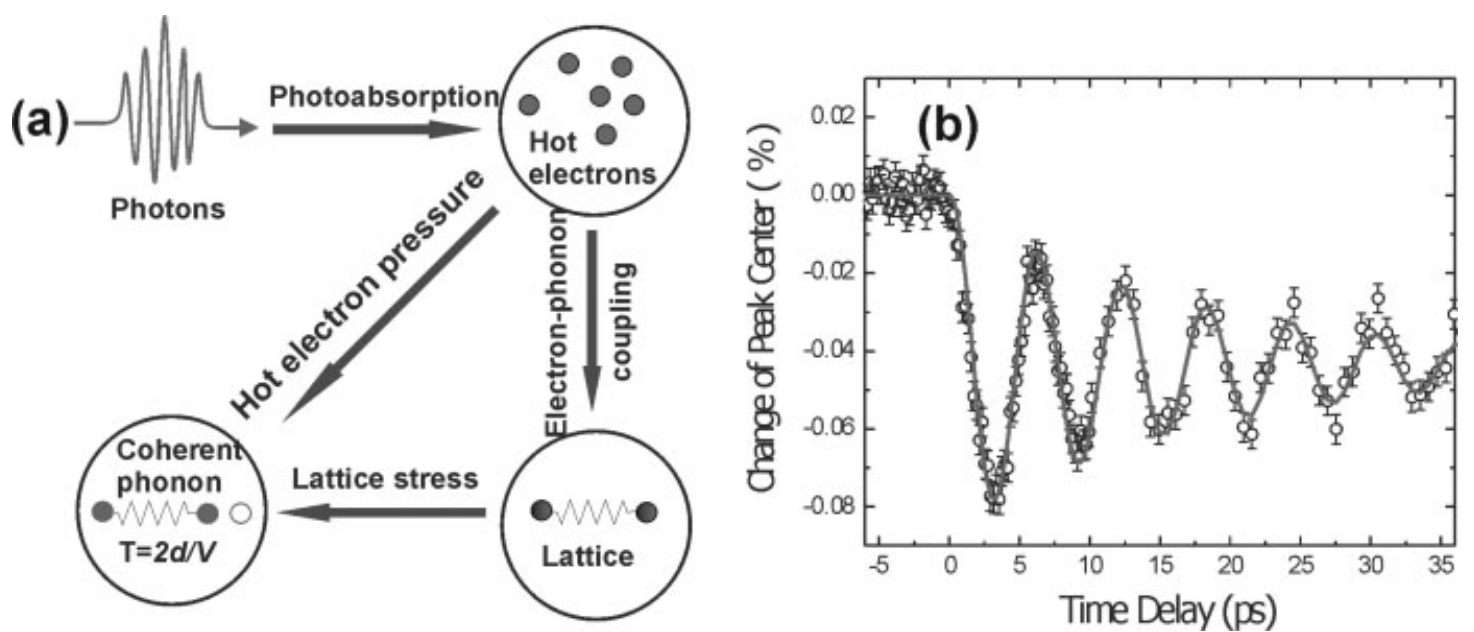

Fig. 12. (a) Coherent phonon generation in nonmagnetic metals. (b) Temporal evolution of coherent phonon amplitude. The open circles are arithmetic averaging of the vibration data of all Bragg peaks. The solid curve is a fit using Eqs. 7 and 10.

quency $\omega_{0}=0.99 \pm 0.01 \mathrm{THz}$ and the damping constant $1 / \beta=15 \pm 1 \mathrm{ps}$, match very well to the coherent lattice vibration data. The fitted value of $\omega_{0}$ is the same as that obtained with a direct Fourier transform of the corresponding vibration data.

The transient stresses from the lattice and the electron heating determined using the above data fitting are plotted in Figure 11(b). These curves show that $\sigma_{1}$ and $\sigma_{\mathrm{e}}$ demonstrate different transient behaviors. $\sigma_{1}$ exhibits a step-function time dependence-the same as that of $\delta T_{1}$-and its asymptotic value determines the new equilibrium lattice position, whereas $\sigma_{e}$ behaves more like a $\delta$ function and only sustains a significant value in the early heating stage before electrons and phonons reach their thermal equilibrium. Importantly, electronic stress $\sigma_{\mathrm{e}}$, with almost equal strength of $\sigma_{1}$ and decaying in about $1.8 \mathrm{ps}$, is a dominant driving force within the first quarter of the coherent phonon period.

To evaluate the role of electronic heating in coherent phonon generation quantitatively, we also fitted the vibration data using only lattice heating as a driving force. The fitting results (with angular frequency $\omega_{0}=$ $1.01 \pm 0.01 \mathrm{THz}$, and damping constant $1 / \beta=16 \pm 1$ ps) are shown as the dashed line in Figure 11(a). Although both fittings conform well to experimental data at delay times longer than $10 \mathrm{ps}$, it is apparent that the fit including both $\sigma_{1}$ and $\sigma_{\mathrm{e}}$ is significantly better than that excluding $\sigma_{\mathrm{e}}$ at time points before $10 \mathrm{ps}$. In particular, the latter creates a significant phase lag of $\sim 18 \pm 5^{\circ}$ with respect to the vibration data [Fig. 12 (a), inset]. These results provide direct and unambiguous evidence that electronic thermal expansion is essential to drive coherent acoustic phonons and in particular plays a dominant role before the first quarter cycle of lattice vibration.

\section{Electronic Grüneisen Parameters in Ferromagnetic Transition Metals}

Electronic thermal expansion in ferromagnetic transition metals and their alloys is responsible for their anomalous magnetovolume properties, such as the Invar effect, and has been a subject of intensive research for many years. At present, a quantitative description of electronic thermal expansion is still incomplete, which can be largely attributed to the lack of a thorough understanding of magnetism in ferromagnetic transition metals at finite temperature (Baberschke et al., 2001). Therefore, the FED study of dynamical behavior of electronic thermal expansion induced by ultrafast optical excitation will provide an alternative approach for gaining important insights into ferromagnetic transition metal magnetism and related physical processes.

The magnitude of electronic thermal expansion is measured with electronic Grüneisen parameter $\gamma_{\mathrm{e}}$, a physical quantity relating the electronic contribution to volume change in response to the variation of electron temperature $\left(T_{\mathrm{e}}\right)$. Conventionally, $\gamma_{\mathrm{e}}$ can be measured only at the extremely low sample temperature of a few tens of Kelvin or less, where electronic thermal expansion becomes significant or comparable to the lattice thermal expansion (Barron et al., 1980). Under this condition, the lattice $\left(\infty T^{4}\right)$ and electronic $\left(\infty T^{2}\right)$ contributions to thermal expansion exhibit welldefined and distinct temperature dependence. Consequently, they can be separated, and both lattice Grüneisen parameter $\left(\gamma_{1}\right)$ and electronic Grüneisen parameter $\left(\gamma_{\mathrm{e}}\right)$ can be directly measured. At such low temperatures, however, ferromagnetic metals become magnetized, and the dimensional changes associated with magnetic ordering start to set in. The magnetic contribution displays the same or similar temperature as that of the electronic contribution to thermal expansion (Barron et al., 1980; White, 1965) and makes the measurement of $\gamma_{\mathrm{e}}$ in many magnetic materials virtually impossible.

The development of FED has changed the view of the outlook on this measurement and provided us with a new method of measuring $\gamma_{\mathrm{e}}$ (Nie et al., 2006). In contrast to the conventional approach of lowering sample temperature to boost $\sigma_{\mathrm{e}}$, a great enhancement of elec- 
tronic thermal stress is realized by transiently heating the conduction electrons to a temperature well above the lattice temperature with femtosecond optical pulses. By directly probing the associated thermal expansion dynamics in real time using FED, the contributions of $\sigma_{\mathrm{e}}$ and $\sigma_{1}$ to thermal expansion can be differentiated in the time domain. Below we show the first accurate measurement of $\gamma_{e}$ in $\mathrm{Al}$ at room temperature using this new approach. We can rewrite the thermal stress Eq. (9) in the following form:

$$
\sigma(t)=A-B \mathrm{e}^{-t / \tau_{\mathrm{e}-\mathrm{ph}}} \quad \text { with } \quad \gamma_{\mathrm{e}}=\gamma_{1}(1-B / A)
$$

Once the values of $A$ and $B$ are determined by fitting the lattice oscillation data, $\gamma_{\mathrm{e}}$ can be calculated by using Eq. (10) for a given $\gamma_{1}$. To enhance the SNR, the average oscillation data of all the Bragg peaks was used, and the fitting results are shown in Figure 12(b). Using the fitted values of $A=0.048 \pm 0.001, B=0.016$ \pm 0.003 , and $\gamma_{1}=2.16$ (Collins et al., 1973), we determined $\gamma_{\mathrm{e}}$ to be $1.4 \pm 0.3$ for $\mathrm{Al}$, where the uncertainty of 0.3 is calculated by convoluting the errors in all the parameters involved in the fitting. This $\gamma_{\mathrm{e}}$ value is in excellent agreement with the low-temperature measurement of $\gamma_{\mathrm{e}}=1.6$ (Collins et al., 1973).

Following the accurate determination of $\gamma_{\mathrm{e}}$ in $\mathrm{Al}$, we carried out the first measurement of electronic Grüneisen parameters in ferromagnetic transition metal Ni. The measurement was conducted using the same procedure and at an elevated sample temperature of $T=$ $680 \mathrm{~K}$, well above the Ni Curie point of $627 \mathrm{~K}$ (Kittel, 1996), to eliminate the complications from the magnetic ordering. Using the Ni lattice Grüneisen parameter $\gamma_{1}=$ 1.9 measured at room temperature (White, 1965), we obtained a $\gamma_{\mathrm{e}}$ value of $1.4 \pm 0.3$ (Wang et al., 2008).

This $\gamma_{\mathrm{e}}$ data has provided new insights regarding the role of the magnetic local moment in electronic thermal expansion of ferromagnetic metals at the paramagnetic state. The $\gamma_{e}$ value of $\mathrm{Ni}$ in a paramagnetic state was calculated by Levy and coworkers using the density functional theory (Levy et al., 1987). In the calculation, they applied the finite temperature linear muffin-tin orbitals band structure method together with the Von Barth-Hedin parameterization (Barth and Hedin, 1972) in the frame of local spin-density approximation for electron correlation potential. It has been shown that local spin-density approximation is a powerful method to study electron correlations, especially in transition metals such as $\mathrm{Ni}$ with itinerant 3d-band electrons (Wang and Callaway, 1977). In the calculation, the contribution of local magnetic moments, which persist in the paramagnetic state of $\mathrm{Ni}$, to thermal expansion was excluded. The $\gamma_{\mathrm{e}}$ value of 1.3 obtained in this calculation is in excellent agreement with our experimental value and indicates that the magnetic local moment, even though it persists in paramagnetic state of $\mathrm{Ni}$, does not contribute significantly in electronic thermal expansion. This result is also supported by calculations based on variational theory (Kakehashi and Samson, 1986). Very recently, we have also conducted measurements of $\gamma_{e}$ at sample temperatures below Curie point and found the value of $\gamma_{\mathrm{e}}$ is increased and approaching its low-temperature value of 2.1 (White, 1965) with decreasing sample temperature.

\section{CONCLUSION}

We have highlighted our development of a tabletop femtosecond electron diffractometer with the capability of detecting submilli-ångström lattice spacing change on a subpicosecond timescale. We applied this new technique to study nondestructive and reversible ultrafast structural dynamics in metals in which both thermal and coherent lattice motions initiated by femtosecond laser pulses have been directly probed in real time. It was shown that, under highly nonequilibrium conditions generated via ultrafast heating, electronic thermal expansion is greatly enhanced and plays an important role in coherent acoustic phonon generation. We also demonstrated a new method of measuring the electronic Grüneisen parameter using FED at and above room temperature. This discovery opens the door to study electronic thermal expansion in ferromagnetic transition metals, which are inaccessible using traditional low-temperature techniques.

\section{ACKNOWLEDGMENTS}

The authors thank Drs. Zhao Hao and Hyuk Park for their contributions in developing our FED setup.

\section{REFERENCES}

Anisimov SI, Kapeliovich BL, Perel'man TL. 1974. Electron emission from metal surfaces exposed to ultrashort laser pulses. Sov Phys JETP 39:375.

Baberschke K, Donath M, Nolting W. 2001. Band-ferromagnetism: ground-state and finite-temperature phenomena. Springer Verlag.

Barron THK, Collins JG, White GK. 1980. Thermal-expansion of solids at low-temperatures. Adv Phys 29:609-730.

Barth UV, Hedin L. 1972. Local exchange-correlation potential for spin polarized case 1. J Phys C: Solid State Phys 5:1629.

Baum P, Yang DS, Zewail AH. 2007. 4D visualization of transitional structures in phase transformations by electron diffraction. Science 318:788-792.

Baum P, Zewail AH. 2006. Breaking resolution limits in ultrafast electron diffraction and microscopy. Proc Natl Acad Sci USA 103:16105-16110

Baum P, Zewail AH. 2007. Attosecond electron pulses for 4D diffraction and microscopy. Proc Natl Acad Sci USA 104:18409-18414.

Bonham RA, Fink M. 1974. High-energy electron scattering. New York: Van Nostrand Reinhold.

Cao J, Hao Z, Park H, Tao C, Kau D, Blaszczyk L. 2003. Femtosecond electron diffraction for direct measurement of ultrafast atomic motions. Appl Phys Lett 83:1044-1046.

Cavalleri AL, Siders CW, Brown FLH, Leitner DM, Toth C, Squier JA, Barty CPJ, Wilson KR, Sokolowski-Tinten K, von Hoegen MH, von der Linde D, Kammler M. 2000. Anharmonic lattice dynamics in germanium measured with ultrafast x-ray diffraction. Phys Rev Lett 85:586-589.

Collins JG, White GK, Swenson CA. 1973. Thermal-expansion of aluminum below $35 \mathrm{~K}$. J Low Temp Phys 10:69-77.

Dantus M, Kim SB, Williamson JC, Zewail AH. 1994. Ultrafast electron-diffraction. 5. Experimental time resolution and applications. J Phys Chem 98:2782-2796.

Dudek RC, Weber PM. 2001. Ultrafast diffraction imaging of the electrocyclic ring-opening reaction of 1,3-cyclohexadiene. J Phys Chem A 105:4167-4171.

Dwyer JR, Hebeisen CT, Ernstorfer R, Harb M, Deyirmenjian VB, Jordan RE, Miller RJD. 2006. Femtosecond electron diffraction: 'making the molecular movie'. Philos Trans R Soc London Ser A 364:741-778.

Eesley GL. 1986. Generation of nonequilibrium electron and lattice temperatures in copper by picosecond laser-pulses. Phys Rev B 33:2144-2151.

Elsayedali HE, Juhasz T, Smith GO, Bron WE. 1991. Femtosecond thermoreflectivity and thermotransmissivity of polycrystalline and single-crystalline gold-films. Phys Rev B 43:4488-4491. 
Gao HX, Peng LM. 1999. Parameterization of the temperature dependence of the Debye-Waller factors. Acta Crystallogr Sect A 55:926-932.

Garrett GA, Albrecht TF, Whitaker JF, Merlin R. 1996. Coherent THz phonons driven by light pulses and the Sb problem: what is the mechanism? Phys Rev Lett 77:3661-3664.

Gedik N, Yang DS, Logvenov G, Bozovic I, Zewail AH. 2007. Nonequilibrium phase transitions in cuprates observed by ultrafast electron crystallography. Science 316:425-429.

Hastings JB, Rudakov FM, Dowell DH, Schmerge JF, Cardoza JD, Castro JM, Gierman SM, Loos H, Weber PM. 2006. Ultrafast timeresolved electron diffraction with megavolt electron beams. Appl Phys Lett 89.

Hebeisen CT, Ernstorfer R, Harb M, Dartigalongue T, Jordan RE, Miller RJD. 2006. Femtosecond electron pulse characterization using laser ponderomotive scattering. Opt Lett 31:3517-3519.

Hodak JH, Henglein A, Hartland GV. 1999. Size dependent properties of Au particles: coherent excitation and dephasing of acoustic vibrational modes. J Chem Phys 111:8613-8621.

Hohlfeld J, Wellershoff SS, Güdde J, Conrad U, Jähnke V, Matthias E. 2000. Electron and lattice dynamics following optical excitation of metals. Chem Phys 251:237-258.

Hostetler JL, Smith AN, Czajkowsky DM, Norris PM. 1999. Measurement of the electron-phonon coupling factor dependence on film thickness and grain size in $\mathrm{Au}, \mathrm{Cr}$, and Al. Appl Opt 38: 3614-3620.

Hubbell JH, Overbo I. 1979. Relativistic atomic form factors and photon coherent scattering cross-sections. J Phys Chem Ref Data 8:69105.

Kakehashi Y, Samson JH. 1986. Evidence of strong electron correlation-effects on thermal-expansion in transition-metals. Phys Rev B 34:1734-1737.

Kittel C. 1996. Introduction to solid state physics. New York: John Wiley \& Sons.

Kuznetsov AV, Stanton CJ. 1994. Theory of coherent phonon oscillations in semiconductors. Phys Rev Lett 73:3243-3246.

Langelaan G, Saimoto S. 1999. Thermal expansion measurement of pure aluminum using a very low thermal expansion heating stage for x-ray diffraction experiments. Rev Sci Instrum 70:3413-3417.

Levy A, Barak G, Ashkenazi J. 1987. Thermodynamics of copper and nickel-band structure effects and their disappearance at hightemperatures. Phys Rev B 35:9474-9480.

Li J, Wang X, Nie S, Clinite R, Cao J. Coherent atomic motion in nano-crystal film, submitted for publication.

Lide DR, editor. 2001. CRC handbook of chemistry and physics, 82nd ed. Boca Raton: Chapman \& Hall/CRC.

Lindenberg AM, Kang I, Johnson SL, Missalla T, Heimann PA, Chang Z, Larsson J, Bucksbaum PH, Kapteyn HC, Padmore HA, Lee RW, Wark JS, Falcone RW. 2000. Time-resolved x-ray diffraction from coherent phonons during a laser-induced phase transition. Phys Rev Lett 84:111-114.

Mourou G, Williamson S. 1982. Picosecond electron-diffraction. Appl Phys Lett 41:44-45.

Nie S, Wang X, Park H, Clinite R, Cao J. 2006. Measurement of the electronic Grüneisen constant using femtosecond electron diffraction. Phys Rev Lett 96:025901.

Nisoli M, DeSilverstri A, Cavalieri A, Malvezzi AM, Stella A, Lanzani G, Cheyssac P, Kofman R. 1997. Coherent acoustic oscillations in metallic nanoparticles generated with femtosecond optical pulses. Phys Rev B 55:13424-13427.

Park H, Hao Z, Wang X, Nie S, Clinite R, Cao J. 2005a. Synchronization of femtosecond laser and electron pulses with subpicosecond precision. Rev Sci Instrum 76:083905.

Park H, Wang X, Nie S, Clinite R, Cao J. 2005b. Direct and real-time probing of both coherent and thermal lattice motions. Solid State Commun 136:559-563.
Park H, Wang X, Nie S, Clinite R, Cao J. 2005c. Mechanism of coherent acoustic phonon generation under nonequilibrium conditions. Phys Rev B 72:100301.

Perner M, Gresillon S, Marz J, von Plessen G, Feldmann J, Porstendorfer J, Berg KJ, Berg G. 2000. Observation of hot-electron pressure in the vibration dynamics of metal nanoparticles. Phys Rev Lett 85:792-795.

Qian BL, Elsayed-Ali HE. 2002. Electron pulse broadening due to space charge effects in a photoelectron gun for electron diffraction and streak camera systems. J Appl Phys 91:462-468.

Reed BW. 2006. Femtosecond electron pulse propagation for ultrafast electron diffraction. J Appl Phys 100:034916.

Reimer L. 1997. Transmission electron microscopy. Berlin: SpringerVerlag.

Richardson CJK, Spicer JB. 2002. Short-time thermoelastic contributions to picosecond-time scale reflectivity measurements of metals. Appl Phys Lett 80:2895-2897.

Schelev MY, Richards MC, Alcock AJ. 1971. Image-converter streak camera with picosecond resolution. Appl Phys Lett 18:354.

Schoenlein RW, Chong HHW, Glover TE, Heimann PA, Leemans WP Padmore HA, Shank CV, Zholents AA, Zolotorev MS, Corlett JS. 2001. Femtosecond x-rays from relativistic electrons: new tools for probing structural dynamics. CR Acad Sci IV Phys 2:1373-1388.

Siwick BJ, Dwyer JR, Jordan RE, Miller RJD. 2002. Ultrafast electron optics: propagation dynamics of femtosecond electron packets. J Appl Phys 92:1643-1648.

Siwick BJ, Dwyer JR, Jordan RE, Miller RJD. 2003. An atomic-level view of melting using femtosecond electron diffraction. Science 302:1382-1385.

Srinivasan R, Lobastov VA, Ruan CY, Zewail AH. 2003. Ultrafast electron diffraction (UED) - a new development for the 4D determination of transient molecular structures. Helv Chim Acta 86:17631838.

Sun CK, Vallée L, Acioli L, Ippen EP, Fujimoto JG. 1993. Femtosecond investigation of electron thermalization in gold. Phys Rev B 48:12365-12368.

Sundström V. 1996. Femtochemistry and femtobiology: ultrafast reaction dynamics at atomic-scale resolution. River Edge, NJ: World Scientific.

Tas G, Maris HJ. 1994. Electron-diffusion in metals studied by picosecond ultrasonics. Phys Rev B 49:15046-15054.

Thomsen C, Strait J, Vardeny Z, Maris HJ, Tauc J, Hauser JJ. 1984 Coherent phonon generation and detection by picosecond lightpulses. Phys Rev Lett 53:989-992.

Wang CS, Callaway J. 1977. Energy-bands in ferromagnetic nickel. Phys Rev B 15:298-306.

Wang X, Nie S, Li J, Clinite R, Wartenbe M, Martin M, Liang W, Cao J. 2008. Electronic Grüneisen parameter and thermal expansion in ferromagnetic transition metal. Appl Phys Lett 92:121918.

Wang XJ, Xiang D, Kim TK, Ihee H. 2006. J Korea Phys Soc 48:390.

White GK. 1965. Thermal expansion of magnetic metals at low temperatures. Proc Phys Soc London 86:159.

Williamson JC, Zewail AH. 1993. Ultrafast electron-diffraction-velocity mismatch and temporal resolution in crossed-beam experiments. Chem Phys Lett 209:10-16.

Williamson S, Mourou G, Li JCM. 1984. Time-resolved laserinduced phase-transformation in aluminum. Phys Rev Lett 52 2364-2367.

Zeiger HJ, Vidal J, Cheng TK, Ippen EP, Dresselhaus G, Dresselhaus MS. 1992. Theory for displacive excitation of coherent phonons. Phys Rev B 45:768-778.

Zewail AH. 2000. Femtochemistry: atomic-scale dynamics of the chemical bond using ultrafast lasers-(Nobel lecture). Angew Chem Int Ed Engl 39:2587-2631.

Zewail AH. 2006. 4D ultrafast electron diffraction, crystallography, and microscopy. Annu Rev Phys Chem 57:65-103. 